\title{
3 Research Square \\ Clinical characteristics and prognostic factors in elderly patients with COVID-19
}

\section{Qin Cheng}

Peking University Third Hospital https://orcid.org/0000-0002-2107-7248

\section{Yixuan Liao}

Beijing Hospital

\section{Yujing Lin}

Peking University Third Hospital

\section{Liyuan Tao}

Peking University Third Hospital

\section{He Wang}

Beijing Hospital

\section{Mohan Li}

Beijing Hospital

\section{Qinggang Ge}

Peking University Third Hospital

\section{Yanming Li}

Beijing Hospital

Ning Shen ( $\nabla$ shenning1972@126.com)

https://orcid.org/0000-0003-2352-0677

\section{Research article}

Keywords: COVID-19, elderly patients, 28-day mortality, length of hospital stay, prognostic factors.

Posted Date: December 11th, 2020

DOI: https://doi.org/10.21203/rs.3.rs-31123/v3

License: (c) (1) This work is licensed under a Creative Commons Attribution 4.0 International License. Read Full License 


\section{Abstract}

Background: The COVID-19 pandemic posed tremendous threats to the world. Elderly patients were among the high-risk population, and apt to experience worse outcomes.

Methods: Elderly patients (age $\geq 60$ years old) were enrolled from January 28 to February 29, 2020, in Tongji Hospital, one of designated COVID-19 medical centers in Wuhan, China. A retrospective study was performed to describe clinical characteristics and outcomes of elderly COVID-19 patients. COX regression was used to analyze predictors for 28-day mortality. Linear regression models were constructed to analyze factors associated with length of hospital stay (LOS).

Result: A total of 186 elderly patients (aged $70.4 \pm 7.1$ years, 95 males (51.6\%)) were enrolled, 120 patients (64.5\%) were severe or critical type, and mortality rate was $16.1 \%$. Patients in non-survival group had a higher smoking rate, more symptoms of dyspnea, lab results indicative of poorer health. Age (HR $1.128,95 \% \mathrm{Cl} 1.066-1.194)$, lymphocyte count (HR 0.261, 95\% Cl 0.073-0.930), LDH (HR 1.003, 95\% Cl 1.002-1.005), procalcitonin (HR 1.061, 95\% Cl 1.002-1.125), and qSOFA (HR 3.162, 95\% Cl 1.646-6.072) were independently associated with 28-day mortality. CURB-65 plus LDH on admission were predictors of mortality by ROC analysis (AUROC $=0.891$ ). Among surviving patients, consolidation on $\mathrm{CTs}$, elevated ferritin level and neutrophil count were associated with increased LOS.

Conclusion: High incidence of comorbidities and mortality were observed in elderly patients. Decreased lymphocyte, older age, higher qSOFA score, procalcitonin and LDH levels were independent factors associated with mortality, CURB-65 plus LDH could be a predictive model of fatal outcome. Consolidation on CTs, elevated ferritin and neutrophil level correlated with increased LOS. Further prospective studies should be performed to test our findings and explore potential treatments.

\section{Background}

In December 2019, a previously unknown kind of pneumonia, emerged and outbroke in Wuhan, China. It was soon confirmed to be an acute respiratory infectious disease and named novel coronavirus disease 2019 (COVID-19) by World Health Organization (WHO). The pathogen virus was named as severe acute respiratory syndrome coronavirus 2 (SARS-CoV-2). COVID-19 was highly contagious and had caused a global pandemic. According to data from WHO, by Nov 20, 2020, fifty-six million cases had been diagnosed with COVID-19 worldwide, with over 1.3 million deaths. The main manifestations of COVID-19 included fever and dry cough, some patients also experienced dyspnea and hypoxemia. Severe cases might quickly progress to acute respiratory distress syndrome (ARDS), septic shock, multi-organ failure, and even death[1].

Elderly patients were among the high-risk group and apt to experience worse outcomes. Higher prevalence of cancer[2], diabetes[3], and shock[4] were observed in elderly patients with COVID-19 from a few pooled studies. According to epidemiological data from China[5], most deaths occurred in patients over age 60 with underlying diseases. There were higher proportions of severe cases and complications 
like ARDS, cardiac injury, or acute kidney injury. Until now, few studies explored prognostic factors of elderly patients. Wang [6] showed that comorbidities including cardiovascular disease, chronic obstructive pulmonary disease (COPD) were predictive of fatal outcomes. Chen[7] illustrated that males, comorbidities, and time from disease onset to hospitalization were associated with death in older patients. But we still needed a predictive tool of mortality in clinical practice, especially early in-hospital assessment. The pandemic consumes huge healthcare resources, especially for elderly patients with long-time hospital stay. Prolonged length of hospital stay (LOS) could lead to in-hospital complications like secondary infection, venous thromboembolism, etc., which also worse the prognosis. But studies on influencing factors of LOS were deficient.

This retrospective study aimed to describe the clinical and treatment courses of elderly COVID-19 patients and explore predictors associated with 28-day mortality and length of hospital stay (LOS).

\section{Methods}

\section{Study design and participants}

Tongji Hospital, one of the largest comprehensive medical centers in Wuhan, was designated as a specific hospital for COVID-19 patients, especially severe patients, during the outbreak. Isolation wards were managed by supporting medical teams from across China under coordination by the government, due to insufficient medical resources.

In this retrospective study, patients were recruited from six isolation wards of Tongji Hospital, which were managed by medical teams from Peking University Third Hospital, Beijing Hospital and their colleagues. From January 28, to February 29, 2020, all consecutive patients over age 60 and confirmed to have COVID-19 were enrolled. According to "the Diagnosis and Treatment Protocol for Novel Coronavirus Pneumonia (Trial Version 7)" by the National Health Commission of China[1], confirmed cases must have one of the following virologic or serological evidences: positive SARS-Cov-2 nucleic acid by real-time fluorescent RT-PCR or positive serum specific IgM and IgG antibodies of SARS-Cov-2. The severity of COVID-19 was classified according to the guidelines as follows: (1) mild type: clinical symptoms were mild without pneumonia, (2) moderate type: fever and other symptoms presented, with pneumonia on chest computed tomography (CT), (3) severe type: any of the following criteria was met: respiratory rate $\geq 30$ per minute, oxygen saturation $\left(\mathrm{S}_{\mathrm{p}} \mathrm{O}_{2}\right) \leq 93 \%$ on room air at rest, arterial partial pressure of oxygen $\left(\mathrm{PaO}_{2}\right) / \mathrm{FiO}_{2}$ ratio $\leq 300 \mathrm{mmHg}$, (4) critical type: any of the following conditions presented: respiratory failure that required mechanical ventilation, septic shock or other organ failure that required ICU monitoring.

\section{Data collection}

Data were collected from electronic medical records by a trained team of physicians. The parameters consisted of demographic, clinical characteristics, comorbidities, laboratory tests, radiological findings, treatment, and outcome. Comorbidities were mainly determined by past medical history, but diabetes was 
also confirmed by hemoglobin $\mathrm{A} 1 \mathrm{c}(\mathrm{HbA} 1 \mathrm{c}) \geq 6.5 \%$. Chronic respiratory diseases (CRD) were confirmed not only by history of COPD, chronic bronchitis, bronchiectasis, and asthma, but also by existence of bilateral emphysema and bullae on chest CTs. Laboratory tests consisted of blood routine, biochemistry, coagulation, cardiac markers, infection-related indexes, and cytokines. Chest CTs were evaluated by two pulmonary specialists separately. The results were described as the number of lobes infiltrated, the distribution pattern (peripheral, random, or diffuse) and characteristics of pulmonary lesions (groundglass opacities, consolidation, patchy shadow, and pleural effusion). CURB-65 (short for assessment of Consciousness, serum $\underline{\text { Urea nitrogen, }}$ Respiratory rate, $\underline{B}$ lood pressure, and age $\geq \underline{65}$ years) and qSOFA (quick Sequential Organ Failure Assessment) were assessed on admission to quickly evaluate severity of disease. Activities of daily living (ADL) were assessed by Barthel Index score on admission for describing functional abilities[8]. Medications, such as antivirals, antibiotics, and corticosteroids, and advanced supportive procedures, such as mechanical ventilation, were recorded.

\section{Outcome}

Follow-up continued for the hospitalized patients until April 4, 2020. The primary measured outcome was survival or death after 28 days. The secondary outcome was LOS until discharge or endpoint of the study (a study flow diagram is shown in Supplement 1). According to the Chinese guidelines[1], patients could be discharged when symptoms improved significantly, with obvious absorption of infiltrates on chest imaging and negative results for at least two consecutive tests of SARS-CoV-2 nucleic acids.

\section{Statistical analysis}

Continuous variables were expressed as mean \pm SD or median (IQR) with or without normal distribution. Categorical variables were reported as frequencies, and percentages. The independent sample $T$ test or the Mann-Whitney Test were used for comparison between continuous data, and the chi-square test was used for comparison between categorical data. Univariate and multivariate Cox regression analysis, and ROC analysis were used to analyze risk factors of 28-day mortality. Spearman correlation test and multivariate linear regression were used to analyze factors associated with LOS. A two-sided $p<0.05$ was considered statistically significant, and IBM SPSS Statistics (version 22.0) was used for all data analyses.

\section{Results}

\section{Demographic data and clinical characteristics}

A total of 186 patients with confirmed COVID-19 were enrolled in the study, $64.5 \%$ were classified as severe or critical type on admission (shown in Table 1). The mean age was $70.4 \pm 7.1$ years old, and 96 patients $(51.6 \%)$ were male. The mortality rate of patients over age 75 was much higher than that of patients aged $60-74(28.9 \%(13 / 45)$ vs $12.1 \%(17 / 141), p=0.008)$. Lower respiratory rates and higher $\mathrm{S}_{\mathrm{P}} \mathrm{O}_{2}$ levels were found among survivors, but peak temperatures were similar between the two groups. Fever (83.3\%), cough (80.6\%), dyspnea (68.3\%) and fatigue (57.0\%) were the most frequent symptoms 
among older adults, while dyspnea was more common among non-survivors ( $93.3 \%$ vs $53.2 \%, p=0.003$ ). Common comorbidities included hypertension (48.9\%), diabetes (32.8\%), chronic respiratory disease (19.4\%), and coronary heart disease (16.7\%). Non-survivors tended to have higher prevalence of comorbidities, without significant differences. More patients in non-survival group had a smoking history ( $30 \%$ vs $14.1 \%, p=0.032$ ). ADL score in non-survival group was lower than survivors ( $58.8 \pm 27.4$ vs 78.6 $\pm 21.7, p=0.001)$. CURB-65 and qSOFA scores were higher in non-survivors.

Table 1. Demographic and clinical characteristics of elderly COVID-19 patients 


\begin{tabular}{|c|c|c|c|c|}
\hline & $\begin{array}{c}\text { Total } \\
(\mathrm{n}=186)\end{array}$ & $\begin{array}{c}\text { Survivors } \\
(\mathrm{n}=156)\end{array}$ & $\begin{array}{c}\text { Non- } \\
\text { Survivors } \\
(\mathrm{n}=30)\end{array}$ & $\mathrm{P}$ value \\
\hline Age, years old & $70.4 \pm 7.1$ & $69.8 \pm 6.4$ & $73.9 \pm 9.4$ & $0.028^{*}$ \\
\hline $60-74$ yrs. $\mathrm{n}(\%)$ & $141(\overline{75} .8)$ & $124(\overline{7} 9.5)$ & $17(56.7)$ & $0.008 *$ \\
\hline$\geq 75$ yrs. & $45(24.2)$ & $32(20.5)$ & $13(43.3)$ & \\
\hline Gender, Male, n (\%) & $96(51.6)$ & $76(48.7)$ & $20(66.7)$ & 0.072 \\
\hline BMI, $\mathrm{kg} / \mathrm{m}^{2}$ & $\begin{array}{c}23.5(22.0 \\
25.3)\end{array}$ & $\begin{array}{c}23.4(21.8, \\
25.2)\end{array}$ & $\begin{array}{c}23.8(23.0 \\
25.8)\end{array}$ & 0.292 \\
\hline \multicolumn{5}{|l|}{ Vital signs on admission } \\
\hline Heart rate, per minute & $92 \pm 16$ & $91 \pm 16$ & $97 \pm 18$ & 0.056 \\
\hline $\mathrm{SBP}, \mathrm{mmHg}$ & $136 \pm 20$ & $137 \pm 19$ & $132 \pm 26$ & 0.362 \\
\hline $\mathrm{DBP}, \mathrm{mmHg}$ & $81 \pm 13$ & $82 \pm 12$ & $81 \pm 17$ & 0.734 \\
\hline $\mathrm{RR}$, per minute & $21(20,24)$ & $20(20,23)$ & $25(21,30)$ & $<0.001 *$ \\
\hline $\mathrm{S}_{\mathrm{P}} \mathrm{O}_{2}, \%$ & $94(90,97)$ & $95(92,98)$ & $82(76,91)$ & $<0.001 *$ \\
\hline Peak temperature, ${ }^{\circ} \mathrm{C}$ & $38.5 \pm 0.7$ & $38.5 \pm 0.7$ & $38.6 \pm 0.7$ & 0.923 \\
\hline \multicolumn{5}{|l|}{ Symptoms, n (\%) } \\
\hline Fever & 155(83.3) & 128(82.1) & $27(90.0)$ & 0.422 \\
\hline Cough & $150(80.6)$ & $123(78.8)$ & $27(90.0)$ & 0.244 \\
\hline Dyspnea & $127(68.3)$ & $99(53.2)$ & $28(93.3)$ & $0.003^{*}$ \\
\hline Fatigue & $106(57.0)$ & $89(57.1)$ & 17(56.7) & 0.969 \\
\hline Poor appetite & $87(46.8)$ & $71(45.5)$ & $16(53.3)$ & 0.432 \\
\hline Expectoration & $103(55.4)$ & $83(53.2$ & $20(66.7)$ & 0.247 \\
\hline Diarrhea & $73(39.2)$ & $61(39.1)$ & $12(40.0)$ & 0.927 \\
\hline Myalgia & $71(38.2)$ & $61(39.1)$ & $10(33.3)$ & 0.551 \\
\hline Nausea or vomiting & $54(29.0)$ & $45(38.8)$ & $9(30.0)$ & 0.899 \\
\hline Headache & $44(23.7)$ & $38(24.4)$ & $6(20.0)$ & 0.607 \\
\hline \multicolumn{5}{|l|}{ Comorbidities, n (\%) } \\
\hline Hypertension & $91(48.9)$ & $75(48.1)$ & $16(53.3)$ & 0.598 \\
\hline Diabetes mellitus & $61(32.8)$ & $48(30.8)$ & $13(43.3)$ & 0.179 \\
\hline CRD & $36(19.4)$ & $29(18.6)$ & $7(23.3)$ & 0.547 \\
\hline CHD & $31(16.7)$ & $26(16.7)$ & $5(16.7)$ & 1.000 \\
\hline Smoking history & $31(16.7)$ & $22(14.1)$ & $9(30)$ & $0.032 *$ \\
\hline \multicolumn{5}{|l|}{ Severity of disease, $\mathrm{n}(\%)$} \\
\hline Moderate & $66(35.5)$ & $66(42.3)$ & $0(0)$ & $<0.001 *$ \\
\hline Severe & $105(56.4)$ & $85(54.5)$ & $20(66.7)$ & \\
\hline Critical & $15(8.1)$ & $5(3.2)$ & $10(33.3)$ & \\
\hline ADL score & $75.4 \pm 23.8$ & $78.6 \pm 21.7$ & $58.8 \pm 27.4$ & $0.001 *$ \\
\hline CURB-65 & $1.20 \pm 0.83$ & $1.04 \pm 0.70$ & $2.03 \pm 0.93$ & $<0.001 *$ \\
\hline qSOFA & $0.52 \pm 0.58$ & $0.43 \pm 0.53$ & $1.00 \pm 0.59$ & $<0.001 *$ \\
\hline \multicolumn{5}{|l|}{ Treatments, n (\%) } \\
\hline \multicolumn{5}{|l|}{ Medications } \\
\hline Antiviral treatment & 172(92.5) & $142(91.0)$ & $30(100)$ & 0.180 \\
\hline Umifenovir & $135(72.6)$ & $111(71.2)$ & $24(80)$ & 0.320 \\
\hline Oseltamivir & $77(41.4)$ & $69(44.2)$ & $8(26.8)$ & 0.074 \\
\hline Lopinavir-Ritonavir & $41(22.0)$ & $37(23.7)$ & $4(13.3)$ & 0.310 \\
\hline Antibiotics & $152(81.7)$ & $122(78.2)$ & $30(100)$ & $0.010 *$ \\
\hline Corticosteroids & $76(40.9)$ & $49(31.4)$ & $27(90)$ & $<0.001 *$ \\
\hline \multicolumn{5}{|l|}{ Supportive treatment, } \\
\hline HFNC & $16(8.6)$ & $5(3.2)$ & $11(36.7)$ & $<0.001 *$ \\
\hline NIV & $31(16.7)$ & $9(5.8)$ & $22(73.3)$ & $<0.001 *$ \\
\hline IMV & $18(9.7)$ & $1(0.6)$ & 17(56.7) & $<0.001 *$ \\
\hline Time from illness onset to admission, & $12(9,16)$ & $13(9,16)$ & $11(7,13.3)$ & 0.039* \\
\hline
\end{tabular}


days

\begin{tabular}{lcccc} 
LOS, days & $25(17,37)$ & $27.5(20.3$, & $11.5(7.8$, & $<0.001^{*}$ \\
Time from illness onset to death or & $39(30,52)$ & $40.5(34$, & $24.5(15.8$, & $<0.001^{*}$ \\
discharge, days & & $54.8)$ & $33)$ & \\
\hline
\end{tabular}

* $p<0.05$. BMI: body mass index, RR: respiratory rate, SBP: systolic blood pressure, DBP: diastolic blood pressure, $\mathrm{S}_{\mathrm{P}} \mathrm{O}_{2}$ : pulse oxygen saturation, $\mathrm{CRD}$ : chronic respiratory diseases, CHD: coronary heart disease, ADL: ability score in daily life, CURB-65: short for assessment of consciousness, serum urea nitrogen, respiratory rate, blood pressure, and age 65 or older, qSOFA: quick Sequential Organ Failure Assessment. HFNC: High-flow nasal cannula oxygen, NIV: Non-invasive ventilation, IMV: Invasive mechanical ventilation, LOS: Length of hospital stay.

\section{Laboratory findings and chest imaging}

On admission, lymphocytopenia (count $<1.0 \times 10^{9} / \mathrm{L}$ ) was found in $58.9 \%$ of patients. As shown in Table 2 , significantly higher neutrophil count or lower lymphocyte levels were found among non-survivors. Concerning infection-related indexes, high-sensitivity C-reactive protein (hsCRP, 95.2 vs $28.3 \mathrm{mg} / \mathrm{L}$ ), procalcitonin ( $0.21 \mathrm{vs} 0.05 \mathrm{ug} / \mathrm{L})$, or ferritin ( $1514.1 \mathrm{vs} 622.6 \mathrm{ug} / \mathrm{L})$ and cytokines, such as IL-2R and IL-6, were significantly elevated in non-survivors. Lower levels of albumin (31.4 vs $33.0 \mathrm{~g} / \mathrm{L}$ ) and elevated levels of aspartate aminotransferase (AST, 44 vs $27 \mathrm{U} / \mathrm{L}$ ), lactate dehydrogenase (LDH, 473 vs $275 \mathrm{U} / \mathrm{L}$ ), and blood urea nitrogen (BUN, 8.0 vs $4.4 \mathrm{mmol} / \mathrm{L}$ ) were observed in non-survival group. Coagulation abnormalities, such as prolonged prothrombin time (PT, 14.9 vs $14.1 \mathrm{~s}$ ) and elevated D-dimer levels (2.72 vs $1.16 \mathrm{ug} / \mathrm{ml}$ ), were more severe among non-survivors. Levels of cardiac markers, such as highsensitivity Troponin I (hsTnl, 16.1 vs $5.3 \mathrm{pg} / \mathrm{ml}$ ) and N-terminal pro-brain natriuretic peptide (NT-proBNP, $616 \mathrm{vs} 224 \mathrm{pg} / \mathrm{ml}$ ), were higher in non-survival group, but only slightly exceeded the normal range. All $p$ values above were $<0.05$.

Most elderly patients had bilateral and multi-lobular involvement, with the median affected lobes reaching to five. Ground-glass opacities were predominant lesions (97.3\%), while patchy shadows and consolidations were seen in $75.8 \%$ and $48.4 \%$ patients. Peripheral distribution was prevalent in both groups, diffuse distribution was more frequent among non-survivors ( $36.7 \%$ vs $22.4 \%, p=0.098$ ). Pleural effusions were seen in $11.3 \%$ patients.

Table 2. Laboratory finding and chest imaging of elderly COVID-19 patients 


\begin{tabular}{|c|c|c|c|c|}
\hline & $\begin{array}{c}\text { Total } \\
(\mathrm{n}=186) \\
\end{array}$ & $\begin{array}{c}\text { Survivors } \\
(\mathrm{n}=156)\end{array}$ & $\begin{array}{c}\text { Non-Survivors } \\
(\mathrm{n}=30)\end{array}$ & $\mathrm{P}$ value \\
\hline \multicolumn{5}{|l|}{ Blood routine } \\
\hline WBC, $10^{9} / \mathrm{L}$ & $5.8(4.6,7.9)$ & $5.6(4.5,7.1)$ & $9.3(5.4,12.1)$ & $<0.001^{*}$ \\
\hline $\begin{array}{l}\text { Neutrophil count, } \\
10^{9} / \mathrm{L}\end{array}$ & $4.3(3.2,6.2)$ & $4.0(3.1,5.4)$ & $8.0(4.2,10.6)$ & $<0.001 *$ \\
\hline $\begin{array}{l}\text { Lymphocyte count, } \\
10^{9} / \mathrm{L}\end{array}$ & $0.93 \pm 0.44$ & $0.99 \pm 0.44$ & $0.66 \pm 0.36$ & $<0.001^{*}$ \\
\hline $\begin{array}{l}\text { Platelet count, } \\
10^{9} / \mathrm{L}\end{array}$ & $227 \pm 92$ & $232 \pm 89$ & $201 \pm 107$ & 0.053 \\
\hline Hemoglobin, g/L & $124(114,136)$ & $122(114,133)$ & $137(119,147)$ & $0.004^{*}$ \\
\hline \multicolumn{5}{|l|}{ Biochemical results } \\
\hline ALT, U/L & $23(15,40)$ & $24(14,39)$ & $23(19,46)$ & 0.183 \\
\hline AST, U/L & $29(20,42)$ & $27(19,40)$ & $44(36,63)$ & $<0.001 *$ \\
\hline Albumin, g/L & $32.5(30.1,35.5)$ & $33.0(30.3,36.0)$ & $31.4(28.3,33.8)$ & $0.005 *$ \\
\hline TBil, umol/L & $10.0(7.4,14.1)$ & $9.9(7.3,14.2)$ & 10.8(8.1, 14.7) & 0.490 \\
\hline LDH, U/L & $286(234,394)$ & $275(227,342)$ & $473(365,628)$, & $<0.001 *$ \\
\hline BUN, mmol/L & $4.6(3.4,6.5)$ & $4.4(3.4,5.8)$ & $8.0(5.5,11.1)$ & $<0.001 *$ \\
\hline Creatinine, umol/L & $70(58,87)$ & $69(58,84)$ & $84(67,104)$ & 0.003* \\
\hline \multicolumn{5}{|l|}{$\begin{array}{l}\text { Infection-related } \\
\text { indexes }\end{array}$} \\
\hline hsCRP, mg/L & $38.8(8.8,82.4)$ & $28.3(6.9,67.2)$ & $95.2(50.9,170.3)$ & $<0.001 *$ \\
\hline Procalcitonin, ug/L & $0.06(0.03,0.14)$ & $0.05(0.03,0.09)$ & $0.21(0.10,0.74)$ & $<0.001 *$ \\
\hline Ferritin, ug/L & $\begin{array}{c}\text { 680.4(418.7 } \\
1278.3)\end{array}$ & $\begin{array}{c}622.6(362.8 \\
1146.0)\end{array}$ & $\begin{array}{c}\text { 1514.1(874.3 } \\
2113.8)\end{array}$ & $<0.001^{*}$ \\
\hline IL-2R, U/ml & $\begin{array}{c}740.0(468.0 \\
1171.5)\end{array}$ & $\begin{array}{c}676.0(443.0 \\
1036.5)\end{array}$ & $\begin{array}{c}1189.5(843.0 \\
1534.5)\end{array}$ & $<0.001^{*}$ \\
\hline IL-6, pg/ml & $17.3(5.8,46.1)$ & $15.3(4.5,39.2)$ & $64.1(16.7,125.5)$ & $<0.001 *$ \\
\hline \multicolumn{5}{|l|}{ Cardiac markers } \\
\hline hsTNI, pg/ml & $6.0(3.6,14.1)$ & $5.3(3.12,9.9)$ & $16.1(6.4,75.2)$ & $<0.001 *$ \\
\hline NT-proBNP, pg/ml & $251(126,588)$ & $224(112,428)$ & $616(227,1513)$ & $<0.001 *$ \\
\hline \multicolumn{5}{|l|}{ Coagulation factors } \\
\hline $\mathrm{PT}$, seconds & $14.1(13.6,14.8)$ & $14.1(13.5,14.6)$ & $14.9(14.2,15.8)$ & $<0.001 *$ \\
\hline APTT, seconds & $40.9 \pm 6.0$ & $40.7 \pm 5.9$ & $41.9 \pm 6.9$ & 0.324 \\
\hline Fibrinogen, g/L & $5.24 \pm 1.45$ & $5.19 \pm 1.37$ & $5.50 \pm 1.79$ & 0.274 \\
\hline D-dimer, ug/ml & $1.32(0.66,2.62)$ & $1.16(0.61,2.05)$ & $2.72(1.69,16.58)$ & $<0.001 *$ \\
\hline \multicolumn{5}{|l|}{ Chest imaging, n (\%) } \\
\hline $\begin{array}{l}\text { Number of affected } \\
\text { lobes }\end{array}$ & $5(5,5)$ & $5(5,5)$ & $5(5,5)$ & 0.181 \\
\hline \multicolumn{5}{|l|}{$\begin{array}{l}\text { Distribution of } \\
\text { infiltrate }\end{array}$} \\
\hline Peripheral & $110(59.1)$ & 93(59.6) & 17(56.7) & 0.763 \\
\hline Random & $30(16.1)$ & $28(17.9)$ & $2(6.7)$ & 0.205 \\
\hline Diffuse & $46(24.7)$ & $35(22.4)$ & $11(36.7)$ & 0.098 \\
\hline $\begin{array}{l}\text { Ground-glass } \\
\text { opacity }\end{array}$ & $181(97.3)$ & $151(96.8)$ & $30(100)$ & 0.706 \\
\hline Consolidation & $90(48.4)$ & $74(47.4)$ & $16(53.3)$ & 0.554 \\
\hline Patchy shadow & $141(75.8)$ & $121(77.6)$ & $20(66.7)$ & 0.202 \\
\hline Pleural effusion & $21(11.3)$ & 19(12.2) & $2(6.7)$ & 0.576 \\
\hline
\end{tabular}

$* \mathrm{p}<0.05$. WBC: count of white blood cell, ALT: alanine aminotransferase, AST: aspartate aminotransferase, TBil: total bilirubin, LDH: lactate dehydrogenase, BUN, blood urea nitrogen, hsCRP, high sensitivity C-reactive protein, IL-2R: 
interlukin-2 receptor, IL-6: interlukin-6, hsTNI: high sensitivity Troponin I, NT-proBNP: N-terminal pro brain natriuretic peptide, PT: prothrombin time, APTT: Activated partial thromboplastin time.

\section{Treatments and outcomes}

As shown in Table 1, one hundred and seventy-two (92.5\%) patients received antiviral treatment, which mainly included umifenovir, oseltamivir, and lopinavir-ritonavir. 81.7\% (152/186) of patients received antibiotics, while systemic corticosteroids were used for $40.9 \%(76 / 186)$ patients. The time from symptom onset to admission in non-survivors was shorter than survivors ( 13 vs 11 days, $p=0.039$ ). Thirty (16.1\%) patients died after 28-day follow-up. Three cases progressed from moderate to severe, while 26 cases progressed from severe to critical, indicating a deterioration rate of $24.8 \%(26 / 105)$ in the severe-type patients. Followed up to the endpoint, in total 150 patients were cured and discharged, while six patients stayed in hospital. One case was still intubated. The median LOS was 27.5 days for survived patients and only 11.5 days for non-survivors.

\section{Prognostic factors of fatal outcomes in elderly patients}

Univariate COX analysis was used to analyze risk factors associated with 28-day mortality. Older age (HR $1.083,95 \% \mathrm{Cl} 1.032-1.136$ ), decreased SPO2 (HR 0.947, 95\% Cl 0.932-0.963), or increased respiratory rate (HR 1.086, 95\% Cl 1.040-1.134) on admission and the symptom of dyspnea (HR 6.819, 95\% Cl 1.62428.625) correlated with an increased likelihood of death. Comorbidities were not predictive of fatal outcomes, but the presence of smoking history was associated with death (HR 2.378, 95\% Cl 1.0875.203). Decreased lymphocyte (HR 0.139, 95\% Cl 0.045-0.427) and elevated infection-related indexes, such as WBC, neutrophil, hsCRP, procalcitonin, ferritin, and cytokines, were predictive of death. As for other laboratory tests, decreased albumin, elevated LDH, BUN or D-Dimer, and prolonged PT were associated with an increased risk of death. CURB-65 (HR 3.525, 95\% Cl 2.343-5.305) and qSOFA (HR $4.262,95 \% \mathrm{Cl} 2.364-7.685$ ) were strong predictors of poor outcomes. All $p$ values above were $<0.05$, details were shown in Table 3.

Table 3. Univariate and multivariate COX analysis of factors associated with 28-day mortality 


\begin{tabular}{|c|c|c|c|c|c|c|}
\hline & \multicolumn{3}{|c|}{ Univariate COX analysis } & \multicolumn{3}{|c|}{ Multivariate COX analysis $\Delta$} \\
\hline & HR & $95 \%$ CI & P value & & $95 \%$ CI & P value \\
\hline Age & 1.083 & $1.032-1.136$ & $0.001^{*}$ & 1.128 & $1.066-1.194$ & $<0.001^{*}$ \\
\hline Gender & 0.514 & $0.241-1.099$ & 0.086 & & & \\
\hline $\mathrm{S}_{\mathrm{P}} \mathrm{O}_{2}, \%$ & 0.947 & $0.932-0.963$ & $<0.001 *$ & & & \\
\hline Respiratory rate & 1.086 & $1.040-1.134$ & $<0.001^{*}$ & & & \\
\hline \multicolumn{7}{|l|}{ Initial Symptom } \\
\hline Fever & 1.861 & $0.565-6.137$ & 0.307 & & & \\
\hline Cough & 2.114 & $0.641-6.968$ & 0.219 & & & \\
\hline Dyspnea & 6.819 & $1.624-28.625$ & $0.009 *$ & & & \\
\hline \multicolumn{7}{|l|}{ Comorbidities } \\
\hline Hypertension & 1.274 & $0.622-2.612$ & 0.508 & & & \\
\hline Diabetes & 1.7 & $0.825-3.503$ & 0.15 & & & \\
\hline CRD & 1.273 & $0.546-2.968$ & 0.576 & & & \\
\hline CHD & 1.04 & $0.398-2.718$ & 0.936 & & & \\
\hline Smoking history & 2.378 & $1.087-5.203$ & $0.030 *$ & & & \\
\hline \multicolumn{7}{|l|}{ Blood routine } \\
\hline WBC, $10^{9} / \mathrm{L}$ & 1.142 & $1.075-1.214$ & $<0.001 *$ & & & \\
\hline Neutrophil, $10^{9} / \mathrm{L}$ & 1.144 & $1.079-1.212$ & $<0.001 *$ & & & \\
\hline Lymphocyte, $10^{9} / \mathrm{L}$ & 0.139 & $0.045-0.427$ & $0.001 *$ & 0.261 & $0.073-0.930$ & $0.038^{*}$ \\
\hline Platelet count, $10^{9} / \mathrm{L}$ & 0.996 & 0.992-1.001 & 0.094 & & & \\
\hline Hemoglobin, $\mathrm{g} / \mathrm{L}$ & 1.013 & $0.993-1.032$ & 0.2 & & & \\
\hline \multicolumn{7}{|l|}{ Biochemical } \\
\hline ALT, U/L & 1.002 & 0.994-1.011 & 0.569 & & & \\
\hline AST, U/L & 1.003 & 0.999-1.007 & 0.113 & & & \\
\hline Albumin, g/L & 0.885 & $0.808-0.970$ & $0.009 *$ & & & \\
\hline $\mathrm{LDH}, \mathrm{U} / \mathrm{L}$ & 1.003 & $1.002-1.004$ & $<0.001 *$ & 1.003 & $1.002-1.005$ & $<0.001 *$ \\
\hline $\mathrm{BUN}, \mathrm{mmol} / \mathrm{L}$ & 1.132 & $1.081-1.186$ & $<0.001 *$ & & & \\
\hline $\mathrm{Cr}, \mathrm{umol} / \mathrm{L}$ & 1.003 & 0.999-1.007 & 0.143 & & & \\
\hline \multicolumn{7}{|l|}{ Coagulation factors } \\
\hline $\mathrm{PT}, \mathrm{s}$ & 1.659 & $1.348-2.041$ & $<0.001 *$ & & & \\
\hline APTT, s & 1.022 & $0.964-1.083$ & 0.46 & & & \\
\hline FIB, $g / L$ & 1.122 & $0.868-1.451$ & 0.379 & & & \\
\hline D-dimer, ug/ml & 1.085 & $1.041-1.130$ & $<0.001 *$ & & & \\
\hline \multicolumn{7}{|l|}{ Cardiac marker } \\
\hline hsTNI, pg/ml & 1.001 & $1.000-1.001$ & 0.075 & & & \\
\hline NT-proBNP, pg/ml & 1 & $1.000-1.000$ & 0.906 & & & \\
\hline \multicolumn{7}{|l|}{ Infection-related indexes } \\
\hline hsCRP, mg/L & 1.01 & $1.006-1.014$ & $<0.001 *$ & & & \\
\hline Procalcitonin, ug/L & 1.079 & $1.033-1.127$ & $0.001 *$ & 1.061 & $1.002-1.125$ & $0.044^{*}$ \\
\hline Ferritin, ug/L & 1.001 & $1.000-1.001$ & $<0.001 *$ & & & \\
\hline $\mathrm{IL}-2 \mathrm{R}, \mathrm{U} / \mathrm{ml}$ & 1 & $1.000-1.001$ & $0.009 *$ & & & \\
\hline IL-6, pg/ml & 1.004 & $1.002-1.006$ & $<0.001 *$ & & & \\
\hline CURB-65 & 3.525 & $2.343-5.305$ & $<0.001 *$ & & & \\
\hline qSOFA & 4.262 & $2.364-7.685$ & $<0.001 *$ & 3.162 & $1.646-6.072$ & $0.001 *$ \\
\hline
\end{tabular}

$* p<0.05 . \mathrm{S}_{\mathrm{P}} \mathrm{O}_{2}$ : pulse oxygen saturation, $\mathrm{CRD}$ : chronic respiratory diseases, CHD: coronary heart disease, WBC: white blood cell, ALT: alanine aminotransferase, AST: aspartate aminotransferase, LDH: lactate dehydrogenase, BUN, blood urea nitrogen, hsTNI: high sensitivity Troponin I, NT-proBNP: N-terminal pro brain natriuretic peptide, PT:

prothrombin time, APTT: Activated partial thromboplastin time, hsCRP, high sensitivity C-reactive protein, IL-2R: interlukin-2 receptor, IL-6: interlukin-6, qSOFA: quick Sequential Organ Failure Assessment, CURB-65: short for assessment of consciousness, serum urea nitrogen, respiratory rate, blood pressure, and age 65 or older. 
$\Delta$ : The following numerical or categorical indexes (16 parameters, all of which were $p<0.05$ ) were selected for a backward stepwise multivariate COX regression analysis: age, $\mathrm{S}_{\mathrm{P}} \mathrm{O}_{2}$, symptom of dyspnea, smoking history, qSOFA, WBC and lymphocyte count, and other hematological biomarkers, like albumin, LDH, BUN, D-Dimer, hsCRP, procalcitonin, Ferritin, IL-2R, and IL-6.

In the multivariate COX regression, age (HR 1.128, 95\% Cl 1.066-1.194), lymphocyte count (HR 0.261, 95\% $\mathrm{Cl}$ 0.073-0.930), LDH (HR 1.003, 95\% Cl 1.002-1.005), procalcitonin (HR 1.061, 95\% Cl 1.002-1.125), and qSOFA (HR 3.162, 95\% Cl 1.646-6.072) were independent risk factors associated with 28-day mortality. According to ROC analysis, the cut-off value of procalcitonin and qSOFA were $0.09 \mathrm{ug} / \mathrm{ml}$ and $0.5 \mathrm{scores}$, which having little clinical significance. The optimal cut-off of LDH, CURB-65 and lymphocyte count were $360.5 \mathrm{U} / \mathrm{L}, 1$ score and $0.665 \times 10^{9} / \mathrm{L}$, with the AUROC being $0.838,0.775,0.720$, respectively. Combined indexes of CURB-65, LDH or lymphocyte count were used to obtain a more accurate prognostic value. CURB-65 combined with LDH (86.7\% sensitivity, 78.9\% specificity) was a stronger predictor of 28-day mortality than other markers $(A U R O C=0.891)$, shown in Figure 1 and Supplement 2, 3.

\section{Predictive factors for the LOS in elderly patients}

The correlations of demographic and clinical characteristics with the LOS were analyzed among the surviving 156 patients, which is shown in Supplement 4. Analysis by the Spearman correlation test, age, gender, main symptoms, and comorbidities had little correlation with LOS $(p>0.05)$. On admission, severity classification of disease $(r=0.334)$ and $\mathrm{S}_{\mathrm{p}} \mathrm{O}_{2}(r=-0.296)$ were associated with the LOS. LDH, hsCRP, neutrophil count and ferritin were positive correlated with the LOS (shown in Figure 2), while lymphocyte count was negative correlated. On chest imaging, more affected lobes $(r=0.270)$, diffuse distribution $(r=0.205)$ and existence of consolidation $(r=0.396)$ increased the LOS. Use of antivirals or steroids, and advanced supportive treatment was associated with an increased LOS. All $p$ values above were $<0.05$.

Parameters correlated with the LOS $(r>0.2$ or $<-0.2, p<0.05)$ were entered into backward linear regression analysis, which were shown in Supplement 5 . Medications and supportive therapy were excluded because the model was of predictive purpose. In the multivariate linear regression, the existence of consolidation on CTs $\left(\beta_{1}=8.611\right)$, elevated ferritin levels $\left(\beta_{2}=0.004\right)$ and neutrophil count $\left(\beta_{3}=0.806\right)$ were associated with an increased LOS.

\section{Discussion}

This was an observational study of elderly COVID-19 patients, in which we successively assessed factors associated with 28-day mortality and length of hospital stay. The patients were enrolled consecutively from January 28 to February 29, which was an intense period of the epidemic in Wuhan, and was relatively representative of the similar situations many countries now faced with. They were carefully treated and followed up until nearly all patients had reached to endpoint of discharge or death. A few studies analyzed characteristics and fatal outcomes of elderly COVID-19 patients[6, 7], but little attention 
was received to LOS, which was also important for prognosis. To our knowledge, this was the first study clarify factors associated with LOS in elderly patients.

According to a nationwide epidemiological data of whole population, $18.5 \%$ of confirmed COVID-19 patients in China were classified as severe/critical type, with a crude fatality rate of $2.3 \%[5]$. In this study, among elderly COVID-19 patients, $64.5 \%$ were classified as severe or critical type, and the mortality rate was $16.1 \%$, which is higher than previous studies. This was not only owing to the characteristics of elderly patients, but also to the fact that Tongji hospital was designated as the center for severe COVID-19 patients in Wuhan. 75.3\% patients had at least one comorbidity, which was a higher rate than that of COVID-19 patients overall[9-12]. $32.8 \%$ of patients were diagnosed with diabetes in this study, which was higher than that in previous data[6, 13]. It should be noted that most studies acquired diabetes information only from the past medical history, which might underestimate of its actual prevalence in COVID-19 patients. In this study, diabetes was also confirmed by $\mathrm{HbA} 1 \mathrm{c} \geq 6.5 \%$ according to the American Diabetes Association 2010 criteria[14, 15]. Hence, 22 more patients were newly found to combined with diabetes. The ADL scores on admission were higher among survivors, which indicated better functional abilities in this group. But standard deviation was relatively large in both groups, limiting the reliability of the result. Mean ADL score among non-survivors was less than 60 , indicating that these patients could not fulfill daily activities and relied highly on caregivers. In this study, the time before admission was longer than that in other large studies[12], which might be attributed to the severe epidemic situation and relatively limited medical resources during this period in Wuhan. It has been reported that time from disease onset to hospitalization was an independent risk factor for mortality[7]. However, in our study, the time before admission in non-survival group was shorter than that of the survival group, possible reason being priority treatment of the more severe patients.

Previous studies had found that patients with COVID-19 were often associated with decreased lymphocyte count and increased inflammatory indicators $[9,16]$. In COVID-19 patients with severe and fatal diseases, biomarkers of inflammation, cardiac and muscle injury, liver function, kidney function and coagulation function were significantly elevated, and IL-6, IL-10, and ferritin were strong predictors for severe disease[17]. In this study, decreased lymphocyte and elevated inflammatory indicators (WBC, neutrophil, hsCRP, ferritin, procalcitonin, IL-2R, and IL-6) occurred more often among non-survivors, while changes in other lab parameters, such as hypoalbuminemia, myocardial injury (hsTnl, NT-proBNP, LDH), liver injury (AST, LDH), acute kidney injury (BUN, creatinine) and coagulopathy (PT, D-Dimer), indicated increased likelihood of multi-organ dysfunction or failure in non-survival group. Increased inflammatory parameters indicated a hyperinflammatory state, which was susceptible to systemic inflammatory syndrome (SIRS) or sepsis. LDH, ferritin, IL-2R, and IL-6 were all significantly elevated in non-survival group, indicating the presence of cytokine storm syndrome[18].

Li et al. reported[19] that incidences of consolidation, crazy-paving patterns, and bronchial wall thickening in severe/critical patients were significantly higher than those in the moderate patients. Another study showed that the median CT score of the mortality group was higher compared with that of the survival group, with higher frequencies of consolidation and air bronchogram[20]. In this study, manifestations on 
CTs could not predict mortality, but might affect the LOS. More affected lobes, diffuse distribution and existence of consolidation indicated severe injury of lungs, which needed longer duration of treatment and LOS.

At present, a few studies had discussed the prognosis of elderly COVID-19 patients[6, 7, 21]. This study found that increased age, LDH, procalcitonin levels, and qSOFA scores and reduced lymphocyte were predictors of death. Even among the older adults, age was still a risk factor of death. Mortality rate was higher in older patients ( $\geq 75$ years) than those aged 60 to 74. LDH existed in the heart, liver, skeletal muscle, and red blood cells, and increased LDH level indicates damage of these tissue or cells. On other hand, LDH was also an inflammatory biomarker and, combined with other factors like ferritin, could be strong discriminators for severe COVID-19 or ARDS[17, 18]. Procalcitonin had emerged as an indicator of bacterial infection and helped initiate or discontinue antibiotic therapy[22, 23]. In elderly patients with community acquired pneumonia, procalcitonin allowed early detection of severe courses and initiation of suitable treatment[24]. The qSOFA score was first used to identify risk of patients with sepsis for a poor outcome, it only required a few parameters and vital signs, which made it easy to operate in isolation wards. Recent study showed that the prognostic performance of qSOFA for community-acquired pneumonia was similar with that of CURB-65[25]. Studies had shown that T lymphocyte reduced in COVID-19[26] and speculated that coronavirus acted directly on ACE2 receptors of lymphocyte to cause damage[27], or through virus-mediated lymphocyte apoptosis by activating inflammatory responses, similar with SARS[28]. Immune system was weakened in patients with severe lymphocytopenia, they were apt to suffer secondary infection and poor prognosis[29]. The optimal cut-off value of procalcitonin or qSOFA had little clinical significance by ROC analysis, making neither of these two indexes ideal indicators of death. The areas under the ROC for LDH, CURB-65 and lymphocyte count were all higher than 0.7 , and LDH was the highest of the single factor index in predicting death. CURB-65 was another useful assessment in predicting 30-day mortality of CAP, it contained five parameters and was easy to operate. But its predictive value in viral pneumonia were relatively limited[30]. CURB-65 plus LDH might be a stronger predictive index of 28-day mortality in elderly COVID-19 patients, and can be easily obtained on admission, with high sensitivity and specificity.

Based on result from a Fangcang shelter hospital, which mainly offered medical care for mild, moderate or asymptomatic COVID-19 patients during the outbreak, patients with fever before admission and bilateral pneumonia had significantly longer LOS[31]. In this study, consolidation of chest CTs, increased ferritin level and neutrophil count were independent predictors of longer LOS. As mentioned above, consolidation on CTs, higher ferritin, and neutrophil were all associated with severity of diseases[17-19]. Severe patients usually needed more advanced treatment and longer recovery times, which were the main reasons for prolonged hospital stay. On the other hand, consolidation indicated filling of airspace on CTs, which was related to hypoxemia and sometimes required mechanical ventilation. Patients with elevated ferritin or neutrophil might have uncontrolled underlying inflammation and need anti-bacterial treatment, which would have prolonged LOS. 


\section{Limitations}

There were several limitations of the study. First, data might be incomplete due to its retrospective design, and we could not testify effectiveness of medication or supportive treatment. Second, it was a singlecenter study with limited number of patients, and Tongji hospital was a designated center mainly for severe COVID-19 patients in Wuhan. Selected bias would be inevitable. Third, due to rare cases nowadays in China, we could not perform an external validation to testify our predictive model.

\section{Conclusion}

High incidence of comorbidities and high mortality rates were seen in elderly COVID-19 patients. Independent risk factors of 28-day mortality included increased age, qSOFA, LDH, procalcitonin, and a decreased lymphocyte count, and CURB-65 plus LDH on admission could be a strong predictive model of mortality. Existence of consolidation on CTs, elevated ferritin and neutrophil level correlated with increased LOS. Our findings helped improve awareness and evaluation of elderly COVID-19 patients, and enhance the treatment. Further prospective, well-controlled studies should be performed to test our findings and explore optimal therapy.

\section{Abbreviations}

LOS: Length of hospital stay.

BMl: body mass index,

RR: respiratory rate,

SBP: systolic blood pressure,

DBP: diastolic blood pressure,

$\mathrm{SpO}_{2}$ : pulse oxygen saturation,

CRD: chronic respiratory diseases,

CHD: coronary heart disease,

ADL: ability score in daily life.

CURB-65: short for assessment of consciousness, serum urea nitrogen, respiratory rate, blood pressure, and age 65 or older,

qSOFA: quick Sequential Organ Failure Assessment.

HFNC: High-flow nasal cannula oxygen, 
NIV: Non-invasive ventilation,

IMV: Invasive mechanical ventilation.

WBC: count of white blood cell,

ALT: alanine aminotransferase,

AST: aspartate aminotransferase,

TBil: total bilirubin,

LDH: lactate dehydrogenase,

BUN, blood urea nitrogen,

hsCRP, high sensitivity C-reactive protein,

IL-2R: interlukin-2 receptor,

IL-6: interlukin-6,

hsTNI: high sensitivity Troponin I,

NT-proBNP: N-terminal pro brain natriuretic peptide,

PT: prothrombin time,

APTT: Activated partial thromboplastin time.

\section{Declarations}

\section{Ethics approval and consent to participate}

The study was approved by the Ethics Committee of Peking University Third Hospital (IRB00006761M2020060), and written informed consent was waived because of its retrospective design.

\section{Consent for publication}

Not applicable.

\section{Availability of data and materials}

The datasets used for the current study are available from the corresponding authors upon reasonable request. 
The authors declare that they have no competing interests

\section{Funding}

Ning Shen is currently receiving a grant (\#2018YFC1311900) from National Key R\&D Program of China. The funding body had no role in the design of the study and collection, analysis, interpretation of data or in writing the manuscript.

\section{Authors' contributions}

NS and YmL contributed to the conception and design of the study. QC contributed to the design of the study, data acquisition, analysis, and interpretation of the results. YXL contributed to the design of the study, analysis, and interpretation. YjL contributed to the design of the study, data acquisition, and interpretation. LT contributed to statistical analysis of the study. HW and ML contributed to data acquisition and analysis. QG contributed to the design of the study. All authors read and approved the final manuscript.

\section{Acknowledgments}

We thank Dr. Yuanwei Fu, Weili Shi and Ye Liu, who collected data throughout the study. They are from Peking University Third Hospital and receive no compensation for their contributions.

\section{References}

1. National Health Commission of P.R.China. Diagnosis and Treatment Protocol for Novel Coronavirus Pneumonia (Trial Version 7). Mar 42020.

2. Desai A, Sachdeva S, Parekh T, Desai R. COVID-19 and Cancer: Lessons From a Pooled MetaAnalysis. JCO Glob Oncol. 2020;6:557-9.

3. Desai R, Singh S, Parekh T, Sachdeva S, Sachdeva R, Kumar G. COVID-19 and diabetes mellitus: A need for prudence in elderly patients from a pooled analysis. Diabetes Metab Syndr. 2020;14(4):6835 .

4. Desai R, Singh S, Parekh T, Sachdeva S, Kumar L, Sachdeva R, et al. COVID-19 and Shock: A Cautionary Tale for Elderly Patients From a Pooled Analysis. Ann Emerg Med. 2020;75(6):789-91.

5. Novel Coronavirus Pneumonia Emergency Response Epidemiology T. [The epidemiological characteristics of an outbreak of 2019 novel coronavirus diseases (COVID-19) in China]. Zhonghua Liu Xing Bing Xue Za Zhi. 2020;41(2):145-51.

6. Wang L, He W, Yu X, Hu D, Bao M, Liu H, et al. Coronavirus disease 2019 in elderly patients: Characteristics and prognostic factors based on 4-week follow-up. J Infect. 2020.

7. Chen T, Dai Z, Mo P, Li X, Ma Z, Song S, et al. Clinical characteristics and outcomes of older patients with coronavirus disease 2019 (COVID-19) in Wuhan, China (2019): a single-centered, retrospective study. J Gerontol A Biol Sci Med Sci. 2020. 
8. Granger CV, Albrecht GL, Hamilton BB. Outcome of comprehensive medical rehabilitation: measurement by PULSES profile and the Barthel Index. Arch Phys Med Rehabil. 1979;60(4):145-54.

9. Zhou F, Yu T, Du R, Fan G, Liu Y, Liu Z, et al. Clinical course and risk factors for mortality of adult inpatients with COVID-19 in Wuhan, China: a retrospective cohort study. Lancet. 2020;395(10229):1054-62.

10. Chen N, Zhou M, Dong X, Qu J, Gong F, Han Y, et al. Epidemiological and clinical characteristics of 99 cases of 2019 novel coronavirus pneumonia in Wuhan, China: a descriptive study. Lancet. 2020;395(10223):507-13.

11. Huang C, Wang Y, Li X, Ren L, Zhao J, Hu Y, et al. Clinical features of patients infected with 2019 novel coronavirus in Wuhan, China. Lancet. 2020;395(10223):497-506.

12. Liang WH, Guan WJ, Li CC, Li YM, Liang HR, Zhao Y, et al. Clinical characteristics and outcomes of hospitalised patients with COVID-19 treated in Hubei (epicenter) and outside Hubei (non-epicenter): A Nationwide Analysis of China. Eur Respir J. 2020.

13. Lian J, Jin X, Hao S, Cai H, Zhang S, Zheng L, et al. Analysis of Epidemiological and Clinical features in older patients with Corona Virus Disease 2019 (COVID-19) out of Wuhan. Clin Infect Dis. 2020.

14. American Diabetes A. Diagnosis and classification of diabetes mellitus. Diabetes Care. 2010;33 Suppl 1:S62-9.

15. Wang L, Gao P, Zhang M, Huang Z, Zhang D, Deng Q, et al. Prevalence and Ethnic Pattern of Diabetes and Prediabetes in China in 2013. JAMA. 2017;317(24):2515-23.

16. Guan WJ, Ni ZY, Hu Y, Liang WH, Ou CQ, He JX, et al. Clinical Characteristics of Coronavirus Disease 2019 in China. N Engl J Med. 2020.

17. Henry BM, de Oliveira MHS, Benoit S, Plebani M, Lippi G. Hematologic, biochemical and immune biomarker abnormalities associated with severe illness and mortality in coronavirus disease 2019 (COVID-19): a meta-analysis. Clin Chem Lab Med. 2020.

18. Henderson LA, Canna SW, Schulert GS, Volpi S, Lee PY, Kernan KF, et al. On the alert for cytokine storm: Immunopathology in COVID-19. Arthritis Rheumatol. 2020.

19. Li K, Wu J, Wu F, Guo D, Chen L, Fang Z, et al. The Clinical and Chest CT Features Associated with Severe and Critical COVID-19 Pneumonia. Invest Radiol. 2020.

20. Yuan M, Yin W, Tao Z, Tan W, Hu Y. Association of radiologic findings with mortality of patients infected with 2019 novel coronavirus in Wuhan, China. PLoS One. 2020;15(3):e0230548.

21. Becerra-Munoz VM, Nunez-Gil IJ, Eid CM, Aguado MG, Romero R, Huang J, et al. Clinical profile and predictors of in-hospital mortality among older patients admitted for COVID-19. Age Ageing. 2020.

22. Huang DT, Yealy DM, Filbin MR, Brown AM, Chang CH, Doi Y, et al. Procalcitonin-Guided Use of Antibiotics for Lower Respiratory Tract Infection. N Engl J Med. 2018;379(3):236-49.

23. Lee SH, Chan RC, Wu JY, Chen HW, Chang SS, Lee CC. Diagnostic value of procalcitonin for bacterial infection in elderly patients - a systemic review and meta-analysis. Int J Clin Pract. 2013;67(12):13507. 
24. Heppner HJ, Bertsch T, Alber B, Esslinger AS, Dragonas C, Bauer JM, et al. Procalcitonin: inflammatory biomarker for assessing the severity of community-acquired pneumonia-a clinical observation in geriatric patients. Gerontology. 2010;56(4):385-9.

25. Tokioka F, Okamoto H, Yamazaki A, Itou A, Ishida T. The prognostic performance of qSOFA for community-acquired pneumonia. J Intensive Care. 2018;6:46.

26. Chen G, Wu D, Guo W, Cao Y, Huang D, Wang H, et al. Clinical and immunological features of severe and moderate coronavirus disease 2019. J Clin Invest. 2020.

27. Xu H, Zhong L, Deng J, Peng J, Dan H, Zeng X, et al. High expression of ACE2 receptor of 2019-nCoV on the epithelial cells of oral mucosa. Int J Oral Sci. 2020;12(1):8.

28. He L, Ding Y, Zhang Q, Che $X, H e ~ Y$, Shen $H$, et al. Expression of elevated levels of pro-inflammatory cytokines in SARS-CoV-infected ACE2+ cells in SARS patients: relation to the acute lung injury and pathogenesis of SARS. J Pathol. 2006;210(3):288-97.

29. Tan L, Wang Q, Zhang D, Ding J, Huang Q, Tang YQ, et al. Lymphopenia predicts disease severity of COVID-19: a descriptive and predictive study. Signal Transduct Target Ther. 2020;5:33.

30. Guo L, Wei D, Zhang X, Wu Y, Li Q, Zhou M, et al. Clinical Features Predicting Mortality Risk in Patients With Viral Pneumonia: The MuLBSTA Score. Front Microbiol. 2019;10:2752.

31. Wu S, Xue L, Legido-Quigley $H$, Khan $M, W u ~ H$, Peng $X$, et al. Understanding factors influencing the length of hospital stay among non-severe COVID-19 patients: A retrospective cohort study in a Fangcang shelter hospital. PLoS One. 2020;15(10):e0240959.

\section{Figures}

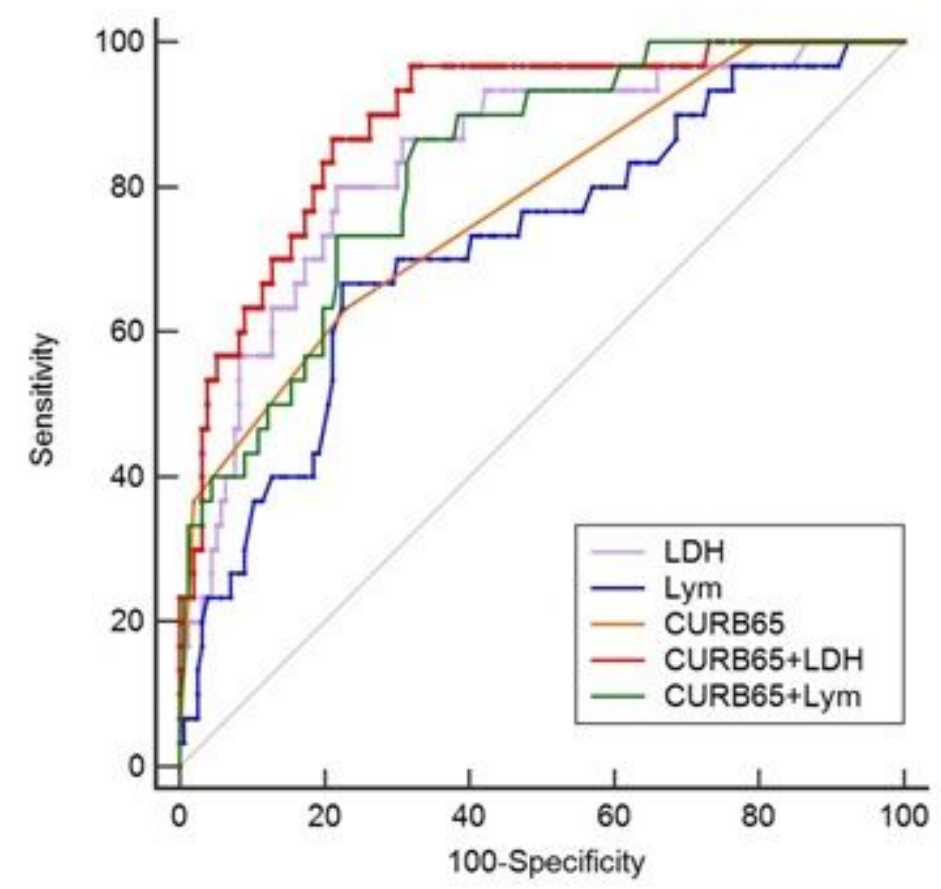

Figure 1 
ROC curve of 28-day mortality. LDH: lactate dehydrogenase, Lym: lymphocyte count, CURB65: short for assessment of consciousness, serum urea nitrogen, respiratory rate, blood pressure, and age 65 or older. AUC of LDH, CURB65, lymphocyte count, CURB65 + LDH, and CURB65 + lymphocyte count were 0.838 , $0.775,0.720,0.891$ and 0.821 , respectively.

A

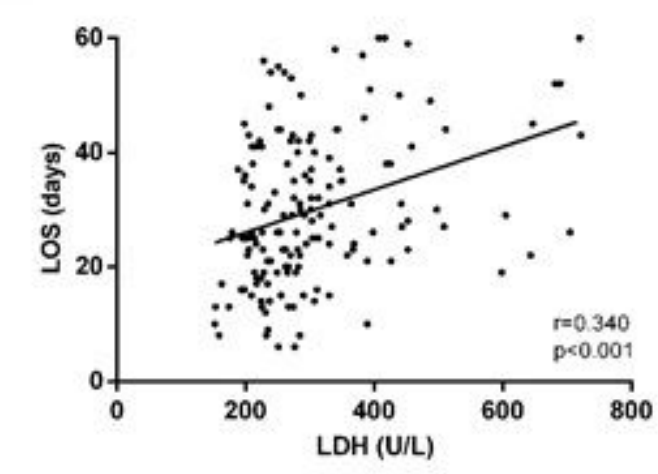

C

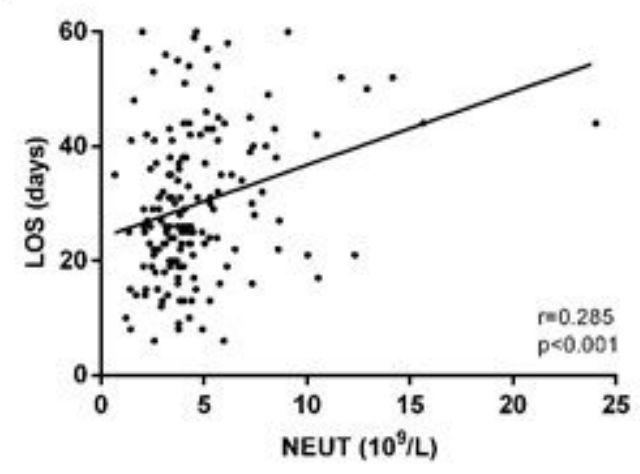

B

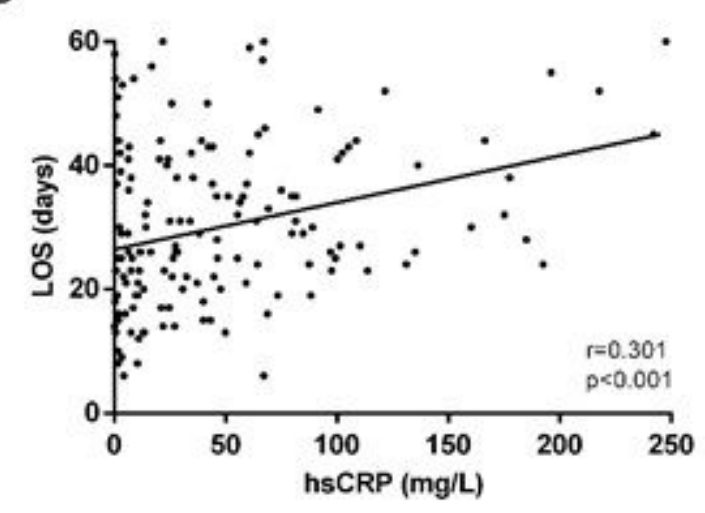

D

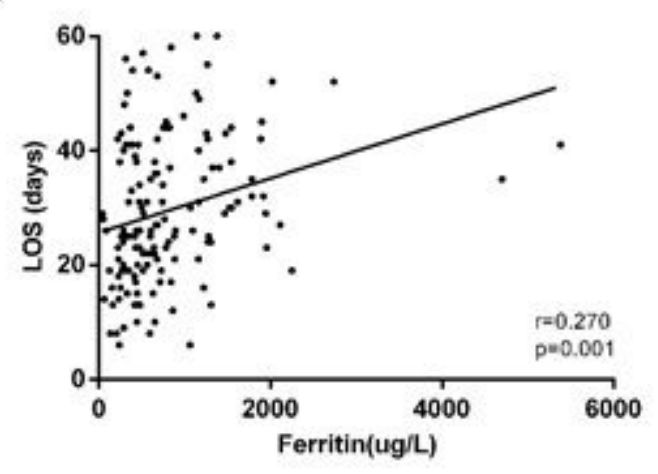

Figure 2

Scatter diagrams, LOS correlated with other indexes. LOS: length of hospital stay. LDH (A. r $=0.340$, $p<0.001)$, hsCRP (B. $r=0.301, p<0.001$ ), neutrophil count (C. $r=0.285, p<0.001)$, and ferritin (D. $r=-0.296$, $\mathrm{p}<0.001)$ were positive correlated with the LOS.

\section{Supplementary Files}

This is a list of supplementary files associated with this preprint. Click to download.

- Supplement1flowchart.tiff 